\title{
¿Espacio de aparición sin dimensión corporal? Identidad $y$ violencia en H. Arendt
}

\author{
Space of Appearance without corporal dimension? \\ Identity and Violence in $\mathrm{H}$. Arendt
}

\begin{abstract}
Resumen: Este artículo analiza la temática del cuerpo humano en la obra de Hannah Arendt, desde la vinculación a su comprensión de la violencia y la identidad individual. $\mathrm{Si}$, por un lado, la filósofa construye una teoría de "lo político" en términos de espacio de aparición y revelación de la identidad personal, el cuerpo, en principio, parece quedar relegado al ámbito privado que ella considera como un espacio de ocultamiento, ligado a la compulsión de la necesidad y la(s) violencia(s) prepolítica(s). Tomando en consideración las tensiones que esta distinción dicotómica plantea, exploraré posibles vías que su misma obra ofrece para trascenderlas y que permiten repensar sus aportaciones a los estudios contemporáneos sobre el cuerpo, así como sus conexiones con la identidad y la violencia.
\end{abstract}

Palabras clave: Arendt, cuerpo, aparición, identidad, violencia, esferas política y prepolítica.

\author{
AGUSTINA VARELA MANOGRASSO*
}

\begin{abstract}
This paper analyzes the theme of the human body in Hannah Arendt's work in its relation to her understanding of violence and individual identity. If, on the one hand, the philosopher builds her theory of "the political" as a space of appearance and disclosure of personal identity, on the other hand, the body seems to be relegated to the private sphere, which is considered as a space of concealment and linked to the compulsion of necessity and prepolitical violence(s). Taking into account the tensions that emerge in this dichotomic distinction, I will explore possible ways that her work gives us to go beyond them and that allows us to rethink Arendtean contributions to contemporary studies of the body, as well as its connections with identity and violence.
\end{abstract}

Keywords: Arendt, appearance, body, identity, violence, political and prepolitical spheres.

Fecha de recepción: 11/06/2016. Fecha de aceptación: 17/07/2016.

* Facultad de Filosofía de la Universidad de Murcia. Becaria del Programa de Formación del Profesorado Universitario (FPU) del Ministerio de Educación, Cultura y Deporte. Referencia AP2012/00635. agustinabelen. varela@um.es agustinavarelamanograsso@gmail.com. Líneas de investigación: Filosofía contemporánea, violencia física y simbólica, identidad personal y colectiva, inclusión y exclusión social.

Referencias: Varela, Agustina, «¿Conformismo o desesperación? Necesidad y violencia en el pensamiento de Hannah Arendt», en: Hernández, P.; Hernández, G.; Juanes, A.; Píriz, C.; Poveda, P.; Rodríguez, M. (coord.), Las Violencias y la Historia, 5 voll., Salamanca, Hergar Ediciones Antema, 2016, pp. 209-227. ISBN:978-84608-9468-1. Varela, Agustina, «De la coacción de la muerte a la coacción de la vida. Dos dimensiones de la violencia en la obra de Hannah Arendt», en: Campillo A., y Manzanero D., (coord.). I Congreso Internacional de la Red Española de filosofía: Los retos de la filosofía en el siglo XXI, 6 voll., Valencia, Facultat de Filosofia i Ciències de l'Educació, Universitat de Vàlencia, 2015, pp. 79-89. ISBN 978-84-370-9680-3 


\section{Introducción}

Toda la propuesta filosófica y política de Hannah Arendt puede ser pensada desde su esfuerzo por distinguir diversas esferas de la vida humana y conceder al terreno de lo político el rango más elevado, donde los humanos pueden ser auténticamente libres sin estar determinados por las coacciones que imponen la necesidad y las relaciones de violencia. Se trataría de un espacio público que, en contraposición al privado, se presenta como lugar de encuentro y de reconocimiento mutuo a través de la acción conjunta, siendo ésta -y el discurso como una forma de acción- la actividad más elevada, que permite a los humanos introducir la novedad en el mundo y revelar su identidad personal.

Sin embargo, la tensión surge al ser presentado como un espacio en que "aparece" un quién, en principio, desligado de su dimensión corporal, que, según Arendt, no puede ser lugar de libertad ni, por tanto, de realización individual precisamente porque pertenece a la dimensión pre-política de la "la simple existencia" sin capacidad de iniciativa ${ }^{1}$. Éste ha sido un punto de confrontación irreconciliable con su pensamiento por parte de muchos intelectuales, sobre todo feministas. Otros, sin embargo, buscaron el modo de "confron-

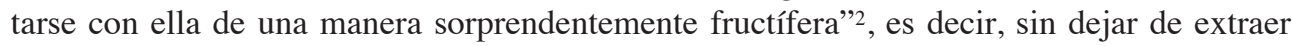
el potencial de sus aportaciones. Y es esta última la línea que pretendo seguir en función de tres objetivos: en primer lugar, analizar brevemente sus nociones de cuerpo e identidad construidas en oposición a la experiencia totalitaria y desde la distinción entre esferas de la vida humana. En segundo lugar, explorar la vinculación que podemos descubrir en sus textos entre necesidad, cuerpo y violencia como amenaza a lo político y contrapuesta a la noción identidad personal. Por último, pretendo indagar en las herramientas que su misma obra puede ofrecer para trascender dicha contraposición.

\section{Espacios de aparición (identidad y revelación) y de ocultación (cuerpo y coacción)}

La distinción entre espacio público y privado recuperada desde otra locución arendtiana que distingue entre "espacio de aparición" (aquello que ha de mostrarse y necesita la presencia de otros) y "espacio de ocultación" (carente de dimensión intersubjetiva y que debe permanecer oculto), permite percibir más claramente la problemática de una esfera en que los individuos "aparecen" unos a otros y que, sin embargo, no parece considerar la dimensión política de sus cuerpos, relegados al ámbito privado. Este esfuerzo por distinguir y jerarquizar ambas esferas está enraizado en su crítica a la tradición del pensamiento occidental, pero más concretamente en la experiencia totalitaria de plena invasión de los espacios tanto público como privado y de la completa indistinción entre uno y otro. Una experiencia vinculada, asimismo, a la reducción de los humanos a la facticidad de sus rasgos biológicos ${ }^{3} \mathrm{o}$ la pertenencia a una determinada "comunidad nacional".

1 Campillo, N., Hannah Arendt: lo filosófico y lo político, Valencia, Universitat de València, 2013, p. 114.

2 Collin, F., «Filosofía y biografía o pensar/contar según Hannah Arendt», en: Segarra, M., (ed.): Praxis de la diferencia. Liberación y libertad, Barcelona, Icaria, 2006, p. 209.

3 "Under a totalitarianism system, all the possibilities for identity are assumed to be already inscribed on the body; the categories of social identity (whether of race, ethnicity, gender, class, or whatever) become embodied absolutes, and the fate of any individual is simply determined by the policies of the all - 
En contraposición al totalitarismo que construyó su sistema anti-político basado en la noción de "naturaleza humana", la filósofa vindica la de "condición humana" que, aun condicionándonos, no nos determina por completo, y un sentido de lo político que no se apoya en las diferencias naturales, sino que es constituido como ámbito de "igualdad artificial" (equality) entre aquellos que se muestran y pueden diferenciarse mediante la palabra y la acción. De modo que el "espacio político de aparición" supone "la presencia de otros que ven lo que vemos y oyen lo que oímos [y que] nos asegura de la realidad del mundo y de nosotros mismos"4. Es decir, la relación interhumana de esta esfera política se presenta como condición para garantizar la realidad misma, pero también -y es lo que en este análisis me interesa resaltar-, para configurar y revelar nuestra propia identidad.

No habría, para la alemana, un "auténtico"s sentido de identidad individual previo al encuentro con los otros en el espacio público-político, porque no habría capacidad de singularización en el ámbito privado. Pero tampoco hacia el interior, algo así como una esencia predeterminada que permita mostrarnos como sujetos únicos. De hecho, según Arendt, desde esta perspectiva interna, todos los humanos somos iguales. Pasiones y emociones son pensadas en gran parte de su obra en términos semejantes a las necesidades fisiológicas, es decir, como parte de la "vida interna" de nuestros cuerpos que nos iguala en la uniformidad de la vida de la especié.

En contraposición a la noción de un "yo (en)cerrado" en sí mismo, Arendt defiende que sólo actuando y hablando aparece la unicidad del sujeto ante la mirada de los otros, que también actúan con nosotros, pero que, a su vez, son espectadores y narradores de nuestras acciones, dándoles sentido una vez concluidas. De este modo, puede decirse que el "yo" arendtiano no es autónomo, ya que depende de los otros para "hacerse visible" y aparecer como unicidad, ni es tampoco un "yo autodeterminado"7, porque la identidad que se revela a los demás permanece oculta al propio actor. En definitiva, para Arendt la identidad individual es una posibilidad que se concreta, aunque nunca de forma conclusiva, en el momento intersubjetivo de la acción y el discurso, y del relato que les dará pleno sentido.

encompassing system in which the body is confined". Moruzzi, N., Speaking Through The Mask: Hannah Arendt and The Politics of Social Identity, Ithaca, Cornell University Press, 2000, p. 115.

4 Arendt, H., La condición humana, Barcelona, Paidós, 1998, p. 60.

5 Hablar de un sentido "auténtico" de identidad, no significa que Arendt no conciba otras identidades configuradas al margen de lo político (como la de los excluidos), o destructoras de lo político (como la de los conformistas o de aquellos que buscan imponer identidades basadas en una única pertenencia, las "identidades asesinas" sobre las que reflexiona Amin Maalouf). Sin embargo, en el caso de las primeras, son invisibilizadas y no son concebidos en su unicidad por los otros. En el caso de las últimas, erradican el espacio de la pluralidad humana donde, y a partir del cual, la singularidad de cada quién puede adquirir realidad para los demás.

6 En este punto reside su crítica a las ciencias del comportamiento y, en concreto, a la psicología, que pretende hallar la identidad individual en el interior de cada cual. Arendt distingue entre "vida del espíritu" y "vida psíquica", concibiendo a la psicología como una "ciencia” centrada en esta última: "[...] no hay sensaciones que correspondan a las actividades mentales; y las sensaciones de la psique, del alma, son sensaciones que experimentamos con los órganos corporales [...] La distinción y la individualización se dan gracias al discurso [...] que no son producto o «símbolos del alma», sino del espíritu [...]”, Arendt, H., La vida del Espíritu, Barcelona, Paidós, 2012, pp. 57-58.

7 " $[\ldots]$ Lo que los sujetos alcanzan al reconocerse mutuamente, según Arendt, no es un mayor autoconocimiento o una mayor autodeterminación, sino el quedar expuestos a la singularidad de los otros y a los riesgos que trae consigo la coexistencia, el ser-en-común en un mundo plural”. Quintana, L., «Identidad sin sujeto: Arendt y el mutuo reconocimiento», Ética \& Política, n 13, 2, 2010, p. 431. 
Ahora bien, en este esquema de la singularización de los humanos aparece la tensión entre la configuración de la identidad personal en el ámbito político de aparición y la desconsideración política de su dimensión corporal, atada al ritmo repetitivo de la naturaleza, y que debe permanecer oculta en el terreno prepolítico de lo privado para no pervertir el sentido de ambas esferas. Se trata de una tensión enraizada en la distinción entre aquello que somos y quiénes somos, y que sitúa al cuerpo en un lugar problemático en las reflexiones arendtianas, en principio, desvinculado de la constitución de la identidad personal.

Ligado al automatismo de la vida biológica y (en)cerrado en una dimensión física de la identidad, que aun definiéndonos como "algo" nada diría de nuestra vida biográfica, el cuerpo formaría únicamente parte de lo que nos ha sido "dado" y, según Arendt, aquello que no podemos poner cuestión, sino aceptar con gratitud ${ }^{8}$. Ser judía y ser mujer, así como el cuerpo físico que había recibido, eran para ella condiciones prepolíticas de su existencia (históricas o naturales), que, si bien decían algo de lo que era, no revelaban "su única y personal identidad" $y$, en ningún caso, debían ser el punto de partida para construir una comunidad política. Si la reticencia arendtiana a concebir una dimensión política del cuerpo parte de su propio encuentro con el nacionalsocialismo, que basó su sistema (anti)político en lo que nos es "dado", despersonalizando a los sujetos y haciendo posible la emergencia de una forma de violencia extrema, es la misma reflexión sobre esta violencia desarrollada plenamente en los campos de exterminio, una vía de análisis que, en mi opinión, permite trascender la perspectiva que ve en su obra un desprecio total hacia el cuerpo humano.

Desde este punto de vista, dos preocupaciones vienen a coincidir en su concepción de cuerpo $^{9}$ : por un lado, aquella que lo concibe como amenaza de lo político y, por tanto, contrapuesto a la configuración de la identidad individual; por otro, una preocupación atenta a la vulnerabilidad de los cuerpos desingularizados -en el contexto totalitario, pero también en el de la sociedad de masas-, y que la llevarían a vindicar su relevancia y protección. Esta ambivalencia de sus reflexiones sobre el cuerpo, ofrece un punto clave desde donde repensar las tensiones y articulaciones entre cuerpo, violencia e identidad en su obra.

\section{El cuerpo como amenaza a lo político: necesidad y violencia}

El primer sentido de esta preocupación se basa en una noción funcional del cuerpo humano ligado a la coacción de las necesidades vitales y, como veremos, a las relaciones prepolíticas de violencia.

8 Para un análisis del cuerpo en la obra arendtiana desde la distinción entre "lo performativo" y "lo constatativo" véase Honig, B., «Toward an Agonistic Feminism: Hannah Arendt and the Politics of Identity», en: Honig, Bonnie (ed.): Feminist Interpretations of Hannah Arendt, Pennsylvania, The Pennsylvania State University Press, 1995, pp. 135-166. Honig critica a Arendt que su gratitud hacia lo dado, lo convirtió en inmodificable y no encontró el modo de repolitizarlo.

9 Tomo como referencia fundamental de este trabajo la siguiente nota a pie de página de Linda Zerilli, desde mi punto de vista, de gran de relevancia para analizar la ambivalencia arendtiana respecto al cuerpo: "Althought Arendt tries to contain the body, she also seeks to protect it. She worries about the vulnerability of bodies in totalitarian and mass societies. Arendt's strategy for shielding the body is crucial to her critique both of the disembodied subject of Enlightenment humanism and of modern mass society [...]”, Zerilli, L., «The Arendtean Body» en: B. Honig (ed.): Feminist Interpretations of Hannah Arendt, Pennsylvania, The Pennsylvania State University Press, 1995, p. 177, (nota 24). 
Esta inquietud arendtiana por el cuerpo -que se convierte en una clara insistencia por contenerlo- aparece ya desarrollada en La condición humana (1958), donde explora las tres actividades de la vita activa dispuestas jerárquicamente en función de sus grados de "aparición" y libertad. Si, por un lado, la actividad laboral (del animal laborans) -centrada en satisfacer las necesidades biológicas-, y el trabajo (del homo faber) -centrado en la producción de objetos-, forman parte de lo prepolítico y estarían (aunque en distintos sentidos) relacionadas significativamente con el cuerpo ${ }^{10}$; la acción, es presentada como la actividad plenamente humana, concebida en términos de libertad y máximo grado de aparición, pero reticente a otorgar relevancia al cuerpo. En esta obra, su desprecio hacia el cuerpo se enmarca en la crítica a las inversiones de esta jerarquía como un largo proceso de despolitización de Occidente, que alcanza su máxima expresión en la época moderna con el ascenso del animal laborans y la ampliación de su "forma de vida" a todas las esferas de la existencia humana, con la consecuente sustitución de la acción por la conducta estandarizada ${ }^{11}$.

No obstante, el tema del cuerpo aparece más concretamente desarrollado en Sobre la revolución (1963). En esta obra, la sustitución de la acción (libertad) por la labor (necesidad) es repensada desde una dura crítica a la Revolución Francesa que, al introducir en su lucha lo que Arendt denomina "la cuestión social" -esto es, la cuestión de la pobreza12 y de las necesidades básicas-, habría despolitizado a la revolución. La preocupación por el cuerpo como amenaza al espacio público aparece aquí reflejada a través de la confrontación directa entre la compulsión del cuerpo físico y la libertad del espacio político ${ }^{13}$, entre cuerpo abyecto homogeneizador y discurso revelador de la identidad. Desde esta perspectiva, parecería que "no sólo para el ciudadano individual, sino para toda la tradición de la acción humana y la historia, el cuerpo presenta una amenaza inminente"14.

Es precisamente este carácter coactivo de lo que se impone directamente sin diálogo, lo que lleva a Arendt a reprimir al cuerpo y sus necesidades en el espacio privado, así como a vincularlo a la violencia prepolítica ${ }^{15}$ en distintos sentidos. En primer lugar, porque al estar sometido a los ciclos repetitivos de la naturaleza y a la coerción que emana de su metabolismo con ésta, puede entenderse como la "violencia" propia de la necesidad que obliga

10 "La filósofa considera que el cuerpo, contrariamente al 'quién', es el agente del proceso vital, bajo los dos aspectos de la fertilidad y el trabajo. Al asegurar el metabolismo de la naturaleza, el cuerpo realiza la reproducción de las necesidades y la satisfacción de las necesidades". Kristeva, J., El genio femenino. 1.Hannah Arendt, Buenos Aires, Paidós, 2000, p. 194. No obstante, es importante señalar que la vinculación entre cuerpo y labor es mucho más estrecha debido a su pleno sometimiento a las necesidades. El trabajo, aún vinculado al cuerpo, supondría un acercamiento a lo que Arendt entiende por libertad (acción), en tanto que mantiene cierto contacto con el mundo a través de los objetos que produce.

11 Se trata de lo que Arendt denominó "el ascenso de lo social”, entendido como "la organización pública del propio proceso de la vida” Arendt, H., La condición humana, op. cit., 56.

12 "La pobreza es algo más que carencia; es un estado de constante indigencia y miseria extrema cuya ignominia consiste en su poder deshumanizante [...]". Arendt, H., Sobre la revolución, Madrid, Alianza, 1988, p. 61.

13 Véase Moruzzi, N., Speaking Through The Mask: Hannah Arendt and The Politics of Social Identity, op. cit., p. 14.

14 Ibid., p. 19. La traducción es mía.

15 Si bien Arendt subraya las diferencias entre necesidad y violencia y está especialmente preocupada en su peligrosa identificación, no niega su estrecha vinculación que aquí propongo repensar a través de la temática del cuerpo. Véase Arendt, H., «Sobre la violencia», en: Crisis de la república, Madrid, Taurus, 1999, pp. 167-175 y Arendt, H., Sobre la revolución, op. cit., pp. 60-114. 
a la subsistencia y niega la libertad. En tanto seres orgánicos, los humanos carecen de la capacidad de interrumpir procesos e iniciar lo inesperado y, por tanto, de la posibilidad de distinguirse mutuamente. Pero es que, además, es en este ámbito de lo privado donde la filósofa sitúa a la violencia instrumental que se introduce entre ellos para dominar, precisamente, la apremiante necesidad.

Así, cuando Arendt se dispone a indagar en la temática del cuerpo humano, lo hace desde la recuperación del modelo griego de lo privado, el oikos, como ámbito jerárquico en el que se ejerce la violencia despótica de unos sujetos sobre otros, para trasladarles la coacción de las necesidades vitales y así poder acceder a la polis libre de violencia. Sin pretender reproducir este modelo, la alemana lo toma como punto de referencia clave para analizar las mutaciones de la época moderna, en la que la vida biológica pasa a convertirse en el mayor bien. Por ello, si bien considera positiva la emancipación de las clases laborantes y la reducción de la violencia premoderna en el ámbito privado y la vida cotidiana, sin embargo, enfatiza los nuevos peligros que introdujo la emancipación de la misma actividad laboral al poner a los humanos bajo el imperio de la necesidad y de violencia que la acompaña. De hecho, fue precisamente el ascenso del humano cuyo cuerpo y necesidades son su realidad dominante, lo que favoreció la preeminencia de una forma de violencia que no es propiamente física y que reduce a los sujetos al "común denominador de la propia vida orgánica" 16 convirtiéndolos en prescindibles.

En este sentido, se puede decir que la temática del cuerpo en su obra entra en conexión con la violencia y la necesidad desde dos dimensiones distintas, pero estrechamente conectadas. Por un lado, el cuerpo es susceptible de una violencia física que lo daña, hiere e, incluso, mata. Violencia que, además, según el análisis arendtiano del oikos griego, es ejercida sobre algunos hombres y mujeres (o clases/grupos sociales enteros) para dominar la necesidad. Por otro lado, hay una dimensión simbólica-existencial de la violencia, que consiste en reducir a los humanos a la única actividad laboral de sus cuerpos.

Precisamente porque para Arendt la revelación de la identidad personal requiere de un espacio político y éste "sólo empieza donde acaba el reino de las necesidades materiales y la violencia física"17, el quién sólo podría revelarse una vez liberado del cuerpo, concebido como lugar de necesidad y susceptible violencia. Habría en este planteamiento un proceso de naturalización y desingularización del cuerpo humano que lo inscriben en el orden de lo que "nos es dado" y "lo general"18. Es así como "el silencio sobre lo que nos ha sido otorgado" se convertiría en su teoría filosófico-política en la prohibición de debatir sobre el cuerpo, así como de sus necesidades, en el espacio político ${ }^{19}$; pero también en un rechazo a considerarlo partícipe indispensable de la configuración de la identidad individual, que acabaría situándolo, como dice Julia Kristeva, “en las antípodas del quién”20.

16 Arendt, H., «La imagen del infierno», en: Arendt, H., Ensayos de comprensión 1930-1954, Madrid, Caparrós, 2005 , p. 246.

17 Arendt, Hannah, ¿Qué es la política?, Barcelona, Paidós, 2007, p. 71. Y, podríamos añadir, las violencias (no propiamente físicas) que atentan contra la posibilidad de singularizarnos y ser reconocidos como unicidad.

18 Kristeva, J., El genio femenino. 1. Hannah Arendt, op. cit., p. 196.

19 "The deep silence about 'what has been given and was not, could not, be made' (...) takes the form of prohibition on speaking about the body in public" [...]". Zerilli, L., «The Arendtean Body», op. cit., 171.

20 Kristeva, J., El genio femenino. 1. Hannah Arendt, op. cit., pp. 195-196. 


\section{El cuerpo como aquello que debemos proteger-cuidar: cuerpo e identidad}

Ahora bien, el espacio arendtiano de aparición en ningún caso puede ser pensado como una esfera compuesta de una multiplicidad de "quienes" descorporizados. El quién soy yo que se revela en presencia de los otros, además de no ser "autónomo" ni ser "esencialoriginario", tampoco es un "yo abstracto - trascendental"21. Por eso, no puede sostenerse que en su teoría filosófico-política el cuerpo humano queda reducido por completo al carácter funcional del proceso vital. Lo que en este punto me interesa explorar es si podemos encontrar en sus reflexiones una dimensión singular del cuerpo, que exigiría repensar su plena despolitización, así como las posibilidades de una repolitización.

Con la mirada puesta en los campos de exterminio, Arendt afirmaba que representaban "la imagen del infierno", donde "morían todos juntos, jóvenes y viejos, débiles y fuertes, enfermos y sanos; y morían no como personas, no como hombres y mujeres, niños y adultos, chicos y chicas, [...] sino rebajados a su mínimo común denominador de la propia vida orgánica, sumidos en el más oscuro y hondo abismo de la igualdad primaria [...] como cosas que no tuvieran cuerpo ni alma, siquiera una fisonomía donde la muerte pudiera estampar su sello"22. Era ésta una violencia de intensidad desconocida que superaba la muerte física y atacaba a la propia unicidad ${ }^{23}$. Se trata de sujetos que habían perdido la posibilidad de aparecer como un quién, pero que morían como si tampoco pudieran preservar el qué del que formaría parte su identidad física.

Es precisamente en este morir indistinto sin siquiera un cuerpo singularizado, sino como mero proceso vital que nos iguala en su "monstruosa igualdad sin fraternidad ni humanidad", donde podemos encontrar una apertura a otra perspectiva arendtiana del cuerpo humano ${ }^{24} \mathrm{y}$ que permite retomar la segunda preocupación a la que aludía al comienzo: el cuerpo no ya como aquello que debemos contener o de lo que debemos liberarnos para acceder a la esfera política, sino como aquello que debemos proteger y cuidar para poder participar en ella. Aparece en este punto una tensión entre una dimensión general y otra singular del cuerpo humano. Aquella que nos desindividualiza y nos convierte en animal laborans, y otra que nos singulariza y, en este sentido, podríamos preguntarnos si, de algún modo, formaría parte de la configuración de nuestra identidad personal.

21 " [...] Arendt evades the body but she does not try to escape it and she even acknowledges pleasure in it. She refuses to embrace the abstract transcendental subject - who is nobody and has no body. In short, Arendt does not deny the subject as embodied, but she does not know quite what to do with the body". Zerilli, L., «The Arendtean Body», op. cit., 175.

22 Arendt, H., «La imagen del infierno», op. cit., p. 246.

23 "El punto decisivo en la argumentación de Arendt, es también el punto más original de su lectura del exterminio. Se trata de la muerte de la unicidad como crimen ontológico primario". Cavarero, A., Horrorismo. Nombrando la violencia contemporánea, Barcelona, Anthropos, 2009, p. 80.

24 No es la única vía para ampliar la comprensión del cuerpo en Arendt. No obstante, es la que me permite repensarlo desde su vinculación a la violencia e identidad. Otra vía es la de la conceptualización de la natalidad. Como afirma F. Collin, no debemos olvidar que Arendt no apoyó su teoría en la mortalidad, sino en la natalidad, que nos remite siempre a otro cuerpo. Véase Collin, F., «Hannah Arendt: la acción y lo dado», en: Segarra, M., (ed.): Praxis de la diferencia. Liberación y libertad, Barcelona, Icaria, 2006, p. 146. 
$\mathrm{Si}$, como mencionaba al comienzo, para Arendt hacia el interior de nuestros cuerpos somos idénticos, es el exterior lo que nos distingue, nos individualiza ${ }^{25} \mathrm{y}$, en este sentido, nos "rescata [...] de la carencia de significado de la vida biológica"26. Desde esta dimensión pública, el cuerpo ya no es concebido en términos de puro automatismo e introspección, o como mero agente de la actividad productiva y reproductiva y, por tanto, como amenaza al espacio de libertad, sino que es percibido como parte ineludible del sujeto político. Aparece en público como un yo encarnado, que ocupa un lugar en el espacio de aparición y que lo hace con una determinada constitución, un color de piel, un sexo, etc.

Ahora bien, al preguntarnos por el alcance de esta singularización, podría considerarse que, aun aceptando un lugar para el cuerpo singularizado en el espacio político, continúa remitiéndonos al qué somos y no al quién. Es decir, a aquello que nos define como algo, pero nada revela de nuestra identidad personal. Se trataría de un espacio de aparición con un cuerpo que, aunque singularizado, sigue siendo objetivado, reducido a "cuerpo visto" y no concebido como "cuerpo vivido" 27 . Sin embargo, desde la perspectiva de la preocupación arendtiana por proteger al cuerpo como singularidad, sería posible trascender la idea de un desprecio total a la dimensión corporal que ya no lo situaría en "las antípodas del quién". La dimensión biológica uniforme del cuerpo no sólo lleva a Arendt a rechazarlo y desplazarlo de lo político. Como mencionaba, el horror ante la reducción humana a esta única dimensión y su muerte (tanto ontológico-existencial como física) sin ningún tipo de particularidad, nos remite a otra perspectiva del cuerpo, que entra en conexión con lo político y con la revelación de la identidad de cada cual. Y es que, como hemos visto, el cuerpo no sólo es vida orgánica, también forma parte de "lo recibido" que para Arendt debemos aceptar con gratitud y de lo que, por tanto, no podemos desligarnos cuando nos convertimos en sujetos políticos. Así, el cuerpo que aparece en la esfera pública a través de sus palabras y acciones, pero que se muestra a los demás asimismo con una forma, color, sexo, etc., lo hace también con la experiencia vivida (de inclusión o exclusión; de reconocimiento o discriminación) que lo atraviesa.

En este sentido, podría decirse, siguiendo a François Collin, que lo dado, y el cuerpo en tanto que forma parte de lo dado, "no es ya el enemigo del que habría que desembarazarse, sino aquello a partir de lo cual y con lo cual se empieza a actuar y a hablar" 28 . Pero, además, puesto que a través de la acción y el discurso nos hacemos propiamente presentes ante los otros, el cuerpo no sólo sería la base desde la que actuamos, sino, y en esto podemos seguir a Margaret Betz Hull, "el vehículo a través del cual el individuo se revela y formula su identidad"29; o mejor, no sólo vehículo, es decir, como medio de las acciones y las palabras, sino más bien como expresión-exhibición que forma parte ineludible del sujeto en acción.

25 "Pienso con frecuencia que interiormente todos los hombres son iguales, tan aproximadamente idénticos como el interior de sus cuerpos. Sólo eso que impulsa a la aparición, o sea, precisamente lo exterior, se distingue, es único, etc.”. Arendt, H., Hannah Arendt. Diario filosófico, 1950-1973, Barcelona, Herder, 2006, Cuaderno XXV [16], p.645.

26 Forti, S., Vida del espíritu y tiempo de la polis, Madrid, Cátedra, 2001, p. 333.

27 Desde esta perspectiva, "el cuerpo es pensado en el ámbito temático del aparecer, pero como cuerpo visto y no como cuerpo vivido (que tiende a dejar de lado en su referencia a Merleau-Ponty)”. Collin, F., «Hannah Arendt: la acción y lo dado», op. cit., p. 130.

28 Collin, F., «Hannah Arendt: la acción y lo dado», op. cit., p. 141.

29 Hull, B., The Hidden Philosophy of Hannah Arendt, New York, Routledge, 2002, p. 165. La traducción es mía. 
En este punto, el cuerpo no aparece ya en su carácter funcional ni meramente vinculado a la coacción propia de la naturaleza, o a la violencia instrumental prepolítica de unos sobre otros para dominar las necesidades de la vida, ni tampoco a la reducción de los humanos al proceso vital que los convierte en prescindibles y susceptibles de una violencia extrema, sino como lugar expresivo de identidad, que debemos salvaguardar para poder comenzar a actuar.

\section{Conclusión}

Como conclusión, se puede decir que no son pocas las tensiones que aparecen en la noción arendtiana de cuerpo humano, sobre todo, al vincularla al tema de la identidad y la violencia. Hay en su obra una ambivalencia no resuelta entre cuerpo coactivo que nos excluye de lo político y cuerpo activo que participa de la acción como máximo grado de aparición y, en este sentido, también de la revelación de la identidad personal.

Se trata de una ambivalencia que, si bien permite ampliar el concepto arendtiano de cuerpo más allá del sentido de "la mera existencia corporal" y vinculado a las relaciones de violencia, sin embargo, no permitiría hablar de su efectiva repolitización, ni tampoco de la del ámbito privado al que suele quedar relegado. Y es que, precisamente porque es el mundo -el espacio inter-esse homines-, la mayor preocupación de Arendt, no llegó a desarrollar un tratamiento del cuerpo que lo integre plenamente en el terreno de lo público, y más que como vehículo de la acción, como (co)implicado en la misma. Además, la distinción entre lo público y lo privado del cuerpo se postula de por sí problemática: no sólo por perder de vista la continuidad entre ambas dimensiones de la existencia humana, sino también porque no permitiría percibirlo como núcleo fundamental de exposición a los demás en toda esfera y, precisamente debido a ello, como encrucijada donde la (des)configuración de la identidad y la posibilidad de las relaciones violentas se encuentran.

No obstante, no podemos perder de vista que la prioridad que Arendt da al "mundo común", es inherente a las posibilidades de exposición, distinción y singularización humana $\mathrm{y}$, aunque no sin tensiones, considero que en este aspecto de su teorización el cuerpo adquiere una relevancia fundamental, todavía poco estudiada. En este sentido, este análisis ha tenido como propósito ir más allá de las críticas que encuentran en sus reflexiones un desprecio total del cuerpo y llevar a cabo una relectura que dé cuenta de aquellas zonas más porosas de su pensamiento, que permiten trascender la aparente rigidez de sus distinciones, abriendo nuevas vías de análisis atentas al potencial de su obra como fuente de inspiración ineludible para repensar las relaciones contemporáneas entre cuerpo, identidad y violencia.

\section{Referencias Bibliográficas}

Arendt, Hannah (1988): Sobre la revolución, Alianza, Madrid.

Arendt, Hannah (1998): La condición humana, Paidós, Barcelona.

Arendt, Hannah (1999): «Sobre la violencia», en Crisis de la república, Taurus, Madrid.

Arendt, Hannah (2005): «La imagen del infierno», en Ensayos de comprensión 1930-1954, Caparrós, Madrid.

Arendt, Hannah (2006): Hannah Arendt. Diario filosófico, 1950-1973, Herder, Barcelona. Arendt, Hannah (2007): ¿Qué es la política?, Paidós, Barcelona. 
Arendt, Hannah (2012): La vida del espíritu, Paidós, Barcelona.

Campillo, Neus (2013): Hannah Arendt: lo filosófico y lo político, Universitat de València, Valencia.

Cavarero, Adriana (2009): Horrorismo. Nombrando la violencia contemporánea, Anthropos, Barcelona.

Collin, Françoise (2006): Praxis de la diferencia. Liberación y libertad, Icaria, Barcelona.

Forti, Simona (2001): Vida del espíritu y tiempo de la polis, Cátedra, Madrid.

Honig, Bonnie (1995): «Toward an Agonistic Feminism: Hannah Arendt and the Politics of Identity», en HONIG, Bonnie (ed.): Feminist Interpretations of Hannah Arendt, The Pennsylvania State University Press, Pennsylvania, pp. 135-166.

Hull, Margaret Betz (2002): The Hidden Philosophy of Hannah Arendt, Routledge, New York.

Kristeva, Julia (2000): El genio femenino. 1.Hannah Arendt, Paidós, Buenos Aires.

Moruzzi, Norma (2000): Speaking Through The Mask: Hannah Arendt and The Politics of Social Identity, Cornell University Press, Ithaca.

Quintana, Laura (2010): «Identidad sin sujeto: Arendt y el mutuo reconocimiento», en Ética \& Política, $\mathrm{n}^{\circ}$ 13, 2, pp. 430-448.

Zerilli, Linda (1995): «The Arendtian Body», en HONIG, Bonnie (ed.): Feminist Interpretations of Hannah Arendt, The Pennsylvania State University Press, Pennsylvania, pp. 167-193. 